\title{
Editorial
}

\section{Surgical quality control - A Herculean task not easily achieved The Dutch gastric trial as a primer}

\author{
Harold J. Wanebo \\ Department of Surgery, Roger Williams Medical Center, 825 Chalkstone Avenue, Providence, Rhode Island 02908, USA
}

In this issue of the Journal, Bonenkamp JJ et al. [1] address quality control issues encountered in the Dutch Gastric Cancer Trial. This unique trial was initiated to address a major and vexing question regarding the reason(s) for the striking differences in survival and outcome among Japanese patients and Western series of patients treated with surgery for gastric cancer. The role of extra-gastric node dissection is touted as a major factor in the improved outcome of the Japanese patients [2]. Historic review of Japanese patients appears to confirm this, as there has been a significant improvement in outcome among Japanese patients over the decades since the initiation of the policy of lymphadenectomy according to the specific dictates for identifying, enumerating, and removing the nodes outlined by the Japanese Research Society for the Study of Gastric Cancer [3]. There is also a striking difference in the outcome of Japanese patients who have surgery for gastric cancer versus patients in Western countries, particularly the United States [4]. Additionally, the mortality and morbidity of gastrectomy is higher overall in the West. Although specialized centers which utilize radical gastrectomy and lymphadenectomy have markedly improved outcome and have reduced mortality and morbidity, their results still fall short of the Japanese results [5]. Japanese surgeons have become so convinced of the value of lymphadenectomy that, ethically, they would be unable to conduct a trial in Japan. The Dutch initiated their formidable trial to compare D1 versus D2 resection after undertaking rigorous credentialing of participating surgeons by requiring the instructing surgeons to be trained in the precise technique of node dissection by Japanese surgeons, and requiring rigorous training and credentialing in the trial. If one compares

Offprint requests to: H.J. Wanebo

Received for publication on Dec. 17, 1998; accepted on Dec. 21, 1998 this effort to the National Surgical Adjuvant Breast and Bowl Project (NSABP) lumpectomy trial for breast cancer [6], in which there was a major effort to instruct the surgeons in the proper performance of local excision of breast cancer, the NSABP effort pales in comparison to the quality control efforts by the Dutch. The effect on morbidity and mortality of this trial and other randomized gastric node dissection trials by the Medical Research Council (MRC) attests to the increased difficulties encountered in adopting the Japanese style of node dissection for Western patients $[7,8]$.

To ensure the distinctiveness of the trial arms, D1 versus D2 lymphadenectomy, the Dutch trialists established a rigorous protocol monitoring, which focused on compliance and contamination, in addition to the training of the participating surgeons. Compliance related to the adequacy of removal of lymph node stations, while contamination referred to lymph nodes detected and removed outside the level of the nodal station required by the protocol. The effect of protocol adherence on complications, mortality, and survival was measured. The overall incidence of noncompliance was $80.6 \%$ for D1 dissections and $81.5 \%$ for D2 dissection. Of note, even the Japanese instructor (overall leader) had a $77.8 \%$ non-compliance rate.

Major non-compliance (more than two nodal stations without a yield) was noted in $15.3 \%$ of the D1 dissections and $25.9 \%$ of D2 dissections. Increasing surveillance reduced major non-compliance from $16.7 \%$ to $14.6 \%$ for D1 dissections and from $35.2 \%$ to $20.0 \%$ for D2 dissections. Contamination was reduced from $31.7 \%$ to $22.8 \%$ for D1 dissection and from $36.6 \%$ to $23.5 \%$ for D2 dissection. Major contamination which was modest, remained unchanged. There was no association between protocol adherence and the occurrence of complications or survival. Increasing the rigor of protocol adherence was not associated with increased nodal recovery, nor did it alter morbidity, mortality, or survival. There are numerous multifactorial causes of non- 
compliance, ranging from site assessment and dissection errors by the surgeon, as well as specimen preparation (node retrieval) and histologic examination (incomplete). All of this attests to the technical difficulties of performing the protocol precisely as specified by the Japanese rules. Causes of contamination were generally related to the surgeons' failure to adhere to the protocol.

\section{Conclusion}

The Dutch Gastric Surgery Trial is a unique effort to compare two surgical modalities which differ in the rigor of performance of D2 versus D1 dissection. The presence of major non-compliance (no identified nodal tissue documented at two or more nodal stations) did not appear to significantly alter the outcome of the protocol or overall nodal removal, complications, and survival. This node dissection trial was organized and executed to the maximum of surgical ability to transplant the Japanese node dissection technique into the Western surgical practice arena and thereby to test the survival benefit of a more comprehensive (Japanese style) D2 dissection versus the Western style D1 dissection. Although there are limitations, as described by the authors, the trial as designed and executed has addressed the challenge of the hypothesis, "Is D2 dis- section superior to D1 dissection?" and we await the longterm results on outcome.

\section{References}

1. Bonenkamp JJ, Hermans J, Sasako M, van de Velde CJH. Quality control of lymph node dissection in the Dutch randomized trial of D1 and D2 dissection for gastric cancer. In Press, Gastric Cancer 1998;1:152-9.

2. Maruyama K, Okbayashi K, Kinoshita T. Progress in gastric cancer surgery and its limits of radicality. World J Surg 1987;11:418-26.

3. Kajitani T. Japanese Research Society for the Study of Gastric Cancer. The general rules for gastric cancer study in surgery and pathology. Jpn J Surg 1981;11:127-45.

4. Wanebo H, Kennedy B, Chmiel J, Steele G, Winchester D, Osteen R. Cancer of the stomach: A patient care study by the American College of Surgeons. Ann Surg 1993;218:583-92.

5. Smith JW, Shiu MH, Kelsey L, Brennan MF. Morbidity of radical lymphadenectomy in the curative resection of gastric carcinoma. Arch Surg 1991;126:1469-73.

6. Fisher B, Redmond C, Poisson R. Eight year results of randomized clinical trial comparing mastectomy and lumpectomy with/ without radiation in treatment of breast cancer. New Engl J Med 1989;320:822-8.

7. Cuschieri A, Fayers P, Fielding J, et al. Postoperative morbidity and mortality after D1 and D2 resections for gastric cancer: Preliminary results of the MRC randomized controlled surgical trial. Lancet 1996;347:995-9.

8. Bonenkamp JJ, Van de Velde CJH, Songun I, Welvaart K, Sasako M, Hermans J, for the Dutch Gastric Cancer Group. Randomized comparison of morbidity after D1 and D2 dissection for gastric cancer in 996 Dutch patients. Lancet 1995;345:745-8. 